\section{Case Reports in Ophthalmology}

Case Rep Ophthalmol 2020;11:516-522

\title{
Recurrent Neuroretinitis: A Unique Presentation of Behçet's Disease in a Child
}

\author{
Gilad Rabina $^{a} \quad$ Gil Amarilyo $^{\mathrm{b}}$ Dinah Zur ${ }^{\mathrm{a}} \quad$ Liora Harel $^{\mathrm{b}}$ \\ Zohar Habot-Wilner ${ }^{a}$ \\ aDepartment of Ophthalmology, Tel Aviv Medical Center, Sackler Faculty of Medicine, \\ Tel Aviv University, Tel Aviv, Israel; ' PPediatric Rheumatology Unit, Schneider Children's \\ Medical Center, Petah Tikva, Sackler Faculty of Medicine, Tel Aviv University, \\ Tel Aviv, Israel
}

\section{Keywords}

Behçet's disease · Neuroretinitis · Uveitis · Vitritis · Retinal lesions

\begin{abstract}
We describe a case of Behçet's disease (BD) in a young child that presented with recurrent neuroretinitis and developed retinal lesions during follow-up. A 4.5-year-old girl presented with fever of $39.5^{\circ} \mathrm{C}$, erythema nodosum in her legs, bilateral knee arthritis, and perineum aphthae. On ocular examination, visual acuity was $20 / 25$ in both eyes. Right eye examination was normal and the left eye (LE) showed mild anterior and intermediate uveitis, normal optic disc, and a macular star appearance. Laboratory workup demonstrated elevated C-reactive protein levels, a normal abdominal ultrasound, and a normal colonoscopy. The patient was diagnosed with BD. One month post initial presentation, the patient presented with visual acuity of finger counting in the LE with significant anterior uveitis, mild intermediate uveitis, and recurrent neuroretinitis. Under treatment of IV methylprednisolone, oral betamethasone, infliximab, and colchicine, a complete systemic remission was noticed, and uveitis became quiescent. On last examination, 4.5 years post first presentation, visual acuity was 20/25 in both eyes and the LE demonstrated a remnant of a juxtafoveal retinal scar. To the best of our knowledge, this is the first case of neuroretinitis presenting as a manifestation of pediatric BD. Ophthalmologists should be aware of these unique manifestations of ocular BD.
\end{abstract}




\section{Case Reports in Ophthalmology}

\section{Introduction}

Behçet's disease (BD) is a chronic, relapsing vasculitis of all vessel sizes and most common in countries along the ancient "Silk Road" [1]. Disease diagnosis is based on clinical finding and there are 17 sets of diagnosis/classification criteria for BD [2]. In pediatric BD, the diagnosis is usually made according to the consensus classification criteria for pediatric Behçet's disease (PEDBD) [3], which contains six categories: recurrent oral aphthosis, genital ulceration or aphthosis, skin involvement (erythema nodosum, acneiform lesions, papulopustular lesions, or skin ulceration), ocular involvement, neurological signs (headaches or papillede$\mathrm{ma}$ ), and vascular signs (vessel thrombosis or aneurysm). A minimum of three signs (each in a distinct category) defines pediatric BD.

Uveitis is the most common ocular manifestation of BD, mainly anterior non-granulomatous, which may be associated with hypopyon. In addition, vitritis, retinal infiltrates, and occlusive vasculitis are signs of posterior segment involvement $[4,5]$.

\section{Case Report}

The patient was a 4.5-year-old girl born in Israel, with a Kurdish, Iraqi, and Yemenite ancestry. Medical family history included fathers' ulcerative colitis and psoriasis. The girl presented with fever of $39.5^{\circ} \mathrm{C}$, erythema nodosum in both legs, bilateral knee arthritis, and perineum aphthae. On ocular examination, BCVA was 20/25 in both eyes. Right eye (RE) examination was normal and the left eye (LE) showed mild anterior uveitis with cells +1 , mild intermediate uveitis with vitreous haze +1 , and a normal optic disc accompanied by a macular star appearance compatible with late-stage neuroretinitis (Fig. 1).

The differential diagnosis at this stage included an infectious disease such as cat scratch disease and a non-infectious disease such as Crohn's disease, sarcoidosis, or BD. But with other systemic findings, differential diagnosis was narrowed to Crohn's disease or BD. Laboratory workup demonstrated leukocytosis of $20 \mathrm{~K}$ with $76 \%$ neutrophils, increased C-reactive protein of $89 \mathrm{mg} / \mathrm{L}$ (normal range 0-5), ESR $84 \mathrm{~mm} / \mathrm{h}$, normal chemistry, normal levels of ACE, negative ANA, normal levels of ASLO, and negative serology for Bartonella henselae. Abdominal ultrasound, colonoscopy, and colon biopsy were all normal. The diagnosis of Crohn's disease was ruled out and the patient was diagnosed with BD according to the PEDBD criteria [3] (four signs - erythema nodosum, arthritis, perineum aphthae, and uveitis). Treatment included naproxen $125 \mathrm{mg}$ BID, colchicine $1 \mathrm{mg}$ QD, betamethasone $1.5 \mathrm{mg}$ QD (tapered down $0.5 \mathrm{mg}$ every 2 weeks), and topical treatment with dexamethasone $0.1 \%$ eye drops. One month post initial presentation, under betamethasone $0.5 \mathrm{mg} \times 1 /$ day, the patient presented with BCVA of 20/30 in the RE and finger counting in the LE. The RE presented with mild intermediate uveitis and the LE presented with significant anterior uveitis (cells +4 , flare +2 ) mild intermediate uveitis (vitreous haze +1 ) and recurrent neuroretinitis. RE ocular coherence tomography (OCT) was normal and LE OCT demonstrated hyperreflective foci in the vitreous compatible with vitritis, subretinal fluid extending between the optic disc and the macula, intra-retinal cysts, and hyperreflective foci compatible with intraretinal exudates. LE ultrasound was performed because of poor posterior pole visualization and demonstrated thickening of posterior coats, fluid in the sub-Tenon space, and exudative retinal detachment (Fig. 2). Because of the acute presentation, treatment with IV methylprednisolone pulse was given, followed by IV methylprednisolone $2 \mathrm{mg} /$ day for 5 days, then oral betamethasone $1.5 \mathrm{mg} \times$ 
2/day, which was slowly tapered. In addition, infliximab $10 \mathrm{mg} / \mathrm{kg}$ at 0,2 , and 6 weeks, then every 4 weeks, was commenced.

Two weeks later, the vitritis resolved and retinal lesions were noticed in the periphery of both eyes and adjacent to the fovea in the LE. Methotrexate $15 \mathrm{mg} / \mathrm{kg}$ was added to the regimen; however, it was stopped after 1 month due to elevated liver enzymes and was switched to azathioprine $30 \mathrm{mg} /$ day. Under this treatment a complete systemic remission was noticed, uveitis became quiescent, and most of the retinal lesions resolved.

On last examination, 4.5 years post first presentation, under treatment with infliximab 5 $\mathrm{mg} / \mathrm{kg}$ every 3 months and colchicine $0.5 \mathrm{mg}$ QD, no systemic findings were noted, BCVA was 20/25 in both eyes, and the LE demonstrated a remnant of a juxtafoveal retinal scar (Fig. 3).

\section{Discussion}

Our patient presented with systemic findings of fever, rash, arthritis, perineum aphthae, and LE panuveitis including a late-stage neuroretinitis. During follow-up, recurrent neuroretinitis developed in the LE with retinal lesions in both eyes. The final diagnosis was BD according to the PEDBD [3] classification. This case presents a unique ocular manifestation of BD in a young child.

The onset age of BD is usually 25-30 years, but it may occur before the age of 16 years in $4-26 \%$ of cases [6-8]. A prospective observational cohort study, including 42 centers from 12 countries, with a total of 230 patients diagnosed with pediatric BD, found that oral aphthosis was the presenting sign for $81 \%$ of patients, mean age at first symptom was $7.4 \pm 4.2$ years (range $0-15.9$ years), and the mean age of onset of uveitis was $10.9 \pm 3.6$ years [9]. In addition, the ocular clinical findings included anterior uveitis in $23.7 \%$ of the patients, posterior uveitis in $21.5 \%$, retinal vasculitis in $9.1 \%$, venous thrombosis in $9.6 \%$, and arterial thrombosis or aneurysm in $1.8 \%$ of the patients [9]. Our patient was young, 4.5 years old at presentation, and presented with anterior and intermediate uveitis, but the most impressive ocular finding at presentation was LE neuroretinitis, a very rare finding in BD, which was described in only two adult BD patients $[10,11]$. Schwartz et al. [10] described a case of a 23-year-old male who was diagnosed with BD with ocular manifestations of bilateral anterior uveitis, intermediate uveitis, neuroretinitis, and a unilateral superior hemi vein occlusion with frosted branch angiitis pattern. Al-Mujaini and Wali [11] reported on a 28-year-old male who was diagnosed with $\mathrm{BD}$ and presented with bilateral frosted branch angiitis and neuroretinitis. Moreover, to date, no case described a recurrent neuroretinitis in a BD patient.

Our patient was treated with systemic corticosteroids and corticosteroid-sparing agents, including methotrexate, azathioprine, and infliximab. Under this treatment, the patient demonstrated a very good response with complete resolution of all systemic manifestations and no active uveitis in a long-term follow-up.

\section{Conclusions}

To the best of our knowledge, this is the first case of neuroretinitis presenting as a manifestation of pediatric BD. In addition, our patient developed recurrent neuroretinitis and retinal lesions during follow-up with a final finding of a macular scar, which is an atypical ocular appearance in BD. Ophthalmologists should be aware of these unique manifestations of ocular BD. 


\section{Case Reports in Ophthalmology}

\section{Statement of Ethics}

Patient's consent has been waived by the Institutional Review Board (IRB) of the Tel Aviv Medical Center, Israel.

\section{Conflict of Interest Statement}

The authors have no financial disclosures.

\section{Funding Sources}

No funding or grant support.

\section{Author Contributions}

Gilad Rabina: writing and revising the manuscript. Gil Amarilyo: revising the manuscript, writing the rheumatology part of the manuscript. Dinah Zur: revising the manuscript, interpretation of OCT and US. Liora Harel: writing the rheumatology part of the manuscript. Zohar Habot: writing and revising the manuscript. All authors approve the final version of the manuscript. All author attests that they meet the current ICMJE criteria for authorship.

\section{References}

1 Mat MC, Sevim A, Fresko I, Tüzün Y. Behçet's disease as a systemic disease. Clin Dermatol. 2014 MayJun;32(3):435-42.

2 Davatchi F, Sadeghi Abdollahi B, Chams-Davatchi C, Shahram F, Shams H, Nadji A, et al. The saga of diagnostic/classification criteria in Behcet's disease. Int J Rheum Dis. 2015 Jul;18(6):594-605.

3 Koné-Paut I, Shahram F, Darce-Bello M, Cantarini L, Cimaz R, Gattorno M, et al.; PEDBD group. Consensus classification criteria for paediatric Behçet's disease from a prospective observational cohort: PEDBD. Ann Rheum Dis. 2016 Jun;75(6):958-64.

4 Tugal-Tutkun I. Behçet's Uveitis. Middle East Afr J Ophthalmol. 2009 Oct;16(4):219-24.

5 Yazici H, Fresko I, Yurdakul S. Behçet's syndrome: disease manifestations, management, and advances in treatment. Nat Clin Pract Rheumatol. 2007 Mar;3(3):148-55.

6 Karincaoglu Y, Borlu M, Toker SC, Akman A, Onder M, Gunasti S, et al. Demographic and clinical properties of juvenile-onset Behçet's disease: A controlled multicenter study. J Am Acad Dermatol. 2008 Apr;58(4):57984.

7 Zouboulis CC, Kötter I, Djawari D, Kirch W, Kohl PK, Ochsendorf FR, et al. Epidemiological features of Adamantiades-Behçet's disease in Germany and in Europe. Yonsei Med J. 1997 Dec;38(6):411-22.

8 Kötter I, Vonthein R, Müller CA, Günaydin I, Zierhut M, Stübiger N. Behçet’s disease in patients of German and Turkish origin living in Germany: a comparative analysis. J Rheumatol. 2004 Jan;31(1):133-9.

9 Koné-Paut I, Shahram F, Darce-Bello M, Cantarini L, Cimaz R, Gattorno M, et al.; PEDBD group. Consensus classification criteria for paediatric Behçet's disease from a prospective observational cohort: PEDBD. Ann Rheum Dis. 2016 Jun;75(6):958-64.

10 Schwartz R, Borok S, Goldstein M, Kesler A, Regev K, Elkayam O, et al. Bilateral Neuroretinitis and a Unilateral Superior Hemivein Occlusion with Frosted Branch Angiitis Pattern Presenting Simultaneously in Behçet's Disease. Case Rep Ophthalmol. 2016 Mar;7(1):141-7.

11 Al-Mujaini A, Wali UK. Frosted branch angiitis, neuroretinitis as initial ocular manifestation in Behçet disease. Indian J Ophthalmol. 2011 May-Jun;59(3):240-1. 
Case Reports in Ophthalmology
Case Rep Ophthalmol 2020;11:516-522 DOI: $10.1159 / 000509158$

(c) 2020 The Author(s). Published by S. Karger AG, Basel www.karger.com/cop

Rabina et al.: Recurrent Neuroretinitis: A Unique Presentation of Behçet's Disease in a Child

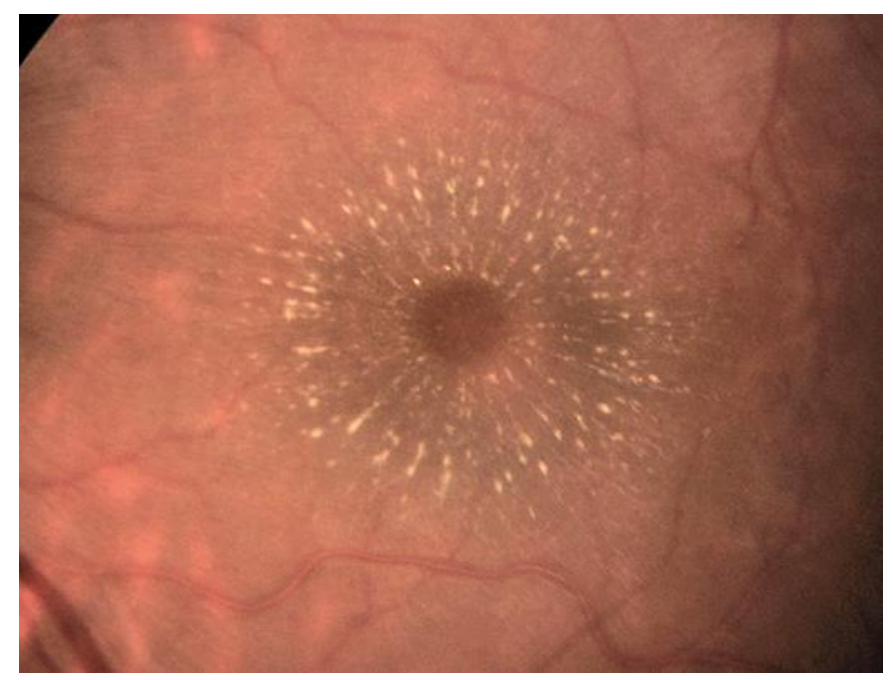

Fig. 1. Fundus photograph of the left eye at presentation showing exudates arranged as a macular star. 

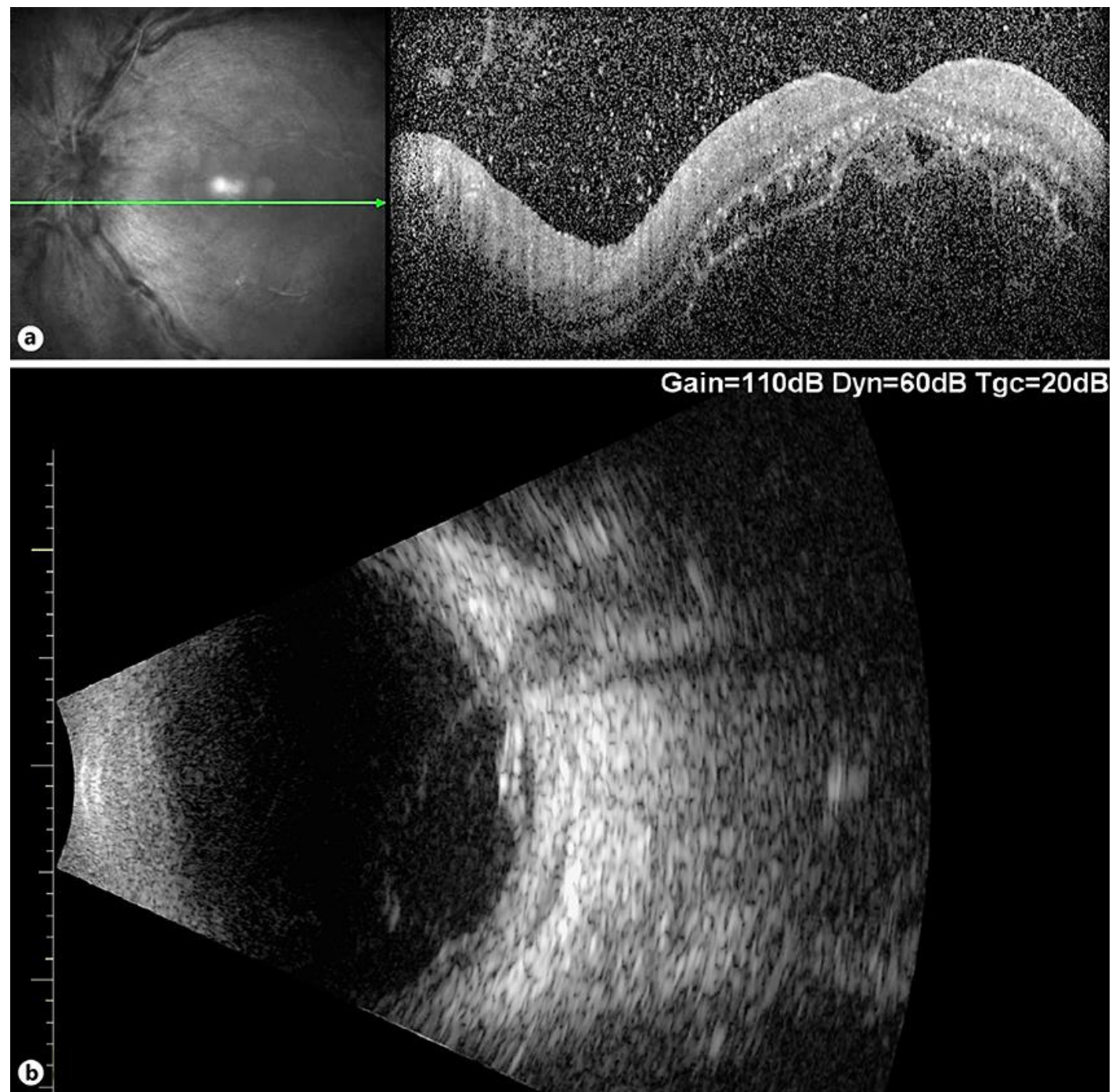

Rabina et al.: Recurrent Neuroretinitis: A Unique Presentation of Behçet's Disease in a Child

Fig. 2. a Horizontal scan encompassing the fovea of the left eye showing hyperreflective foci in the vitreous compatible with vitritis, subretinal fluid extending between the optic disc and the macula, thickening of the retinal nerve fiber layer correlating with optic nerve edema, intra-retinal cystoid hydration, and hyperreflective foci compatible with exudates at the level of the outer plexiform layer. $\mathbf{b}$ B-scan ultrasound showing thickening of posterior coats, fluid in the sub-Tenon space, and exudative retinal detachment. 

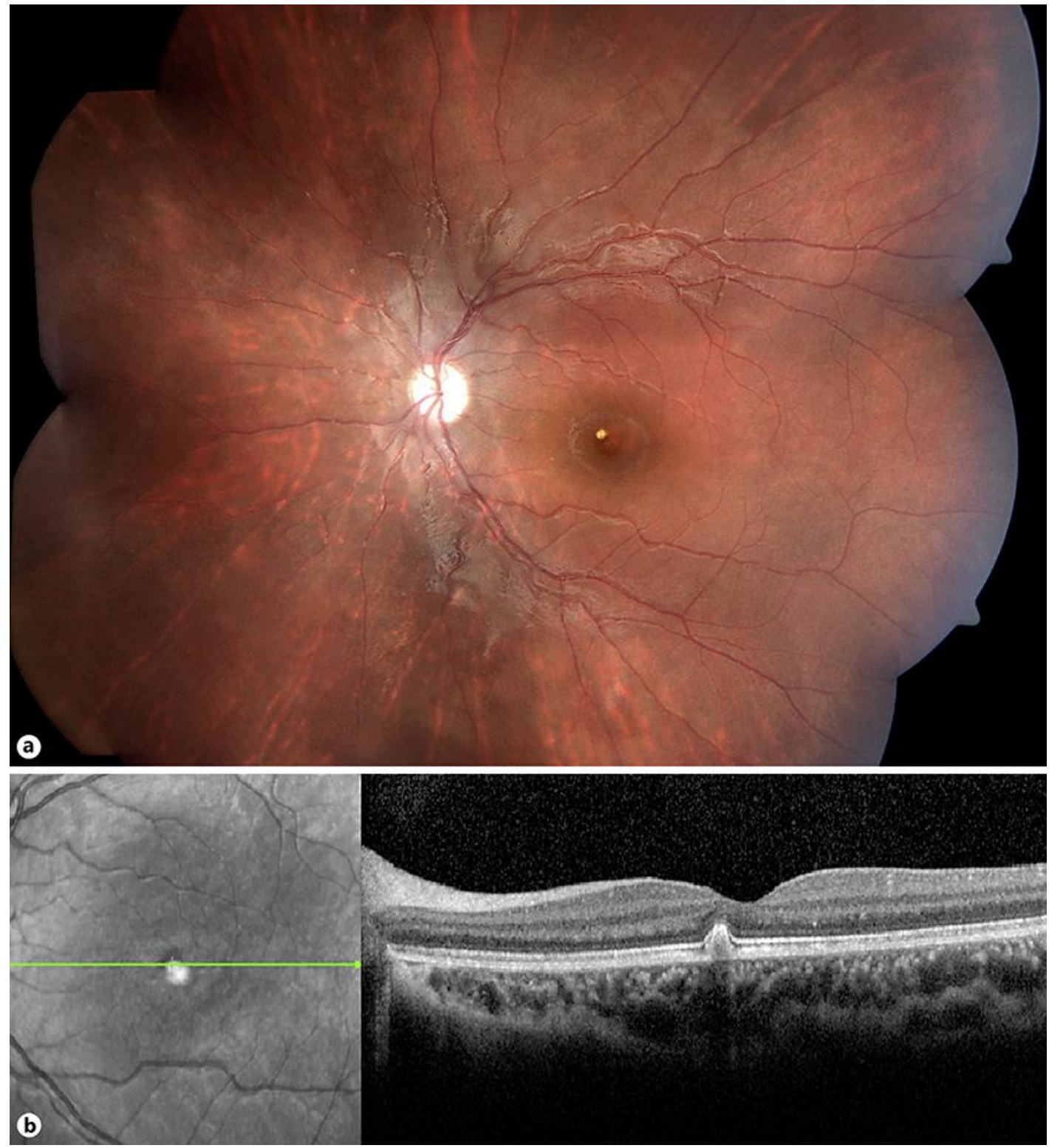

Rabina et al.: Recurrent Neuroretinitis: A Unique Presentation of Behçet's Disease in a Child

Fig. 3. Follow up 4.5 years after initial presentation. a Fundus photograph showing a remnant of a juxtafoveal retinal scar. To mention, although normal, the optic disc appears pale due to overexposure of the fundus photograph. b Horizontal OCT scan showing normal foveal contour without subretinal or intraretinal fluid. Nasal to the fovea hyperreflective thickening of the RPE and irregularity of the external layers, compatible with a retinal scar seen clinically. 